\title{
Supplementary Information: Compact cavity-enhanced single-photon generation with hexagonal boron nitride
}

\author{
Tobias Vogl, ${ }^{1}$ Ruvi Lecamwasam, ${ }^{1}$ Ben C. Buchler, ${ }^{1}$ Yuerui Lu, ${ }^{2}$ and Ping Koy Lam ${ }^{1}$ \\ ${ }^{1}$ Centre for Quantum Computation and Communication Technology, \\ Department of Quantum Science, Research School of Physics and Engineering, \\ The Australian National University, Acton ACT 2601, Australia \\ ${ }^{2}$ Centre for Quantum Computation and Communication Technology, \\ Research School of Electrical, Energy and Materials Engineering, \\ The Australian National University, Acton ACT 2601, Australia
}

\section{CONTENTS}

\begin{tabular}{lr}
\hline S1. Hemisphere fabrication & S2
\end{tabular}

\begin{tabular}{lr}
\hline S2. Spin speed curves PDMS solution & S2 \\
\hline
\end{tabular}

\begin{tabular}{lr}
\hline S3. Photographs of the device & S4
\end{tabular}

\begin{tabular}{lr}
\hline S4. Transverse mode spacing & S4
\end{tabular}

$\begin{array}{lr}\text { S5. FDTD simulations } & \text { S5 }\end{array}$

\begin{tabular}{lr}
\hline S6. QKD simulations & S5
\end{tabular}

This Supplementary Information contains 7 pages and 5 figures. 


\section{S1. HEMISPHERE FABRICATION}

The borosilicate glass substrates (Fisherbrand) have been coated with nominal $100 \mathrm{~nm}$ gold using electron-beam thermal evaporation. During the milling the edges of the substrate (or rather the conductive coating) have been grounded using copper tape. This prevents substrate charging effects, where the ion beam is deflected as more and more charge carriers accumulate in the insulating glass. The $\mathrm{Ga}^{+}$ions are accelerated with a voltage of $30 \mathrm{kV}$, at which our FIB (FEI Helios 600 NanoLab) has sub-nm resolution. We limited the current to $\leq 0.28 \mathrm{nA}$, this way there were no charging effects for milling times of $6 \mathrm{~min}$ or less. During the milling process we add $\mathrm{I}_{2}$-gas, which ensures a smooth surface. The dose rate is encoded in the RGB color of a hemispherical pixel map. The dose rate to RGB value was carefully calibrated, however, we note that this is strongly dependent on the material and conductive coating as well as the state of the ion source. For the calibration we milled squares with edge lengths of $10 \mu \mathrm{m}$ and measured the resulting depth as a function of pixel value. Figure S1(a) shows the trace of the depth scanned through a number of squares. After the milling the layer of gold is dissolved in a custom-made potassium iodide solution $\left(\mathrm{KI}: \mathrm{I}_{2}: \mathrm{H}_{2} \mathrm{O}\right.$ with ratio 4:1:40 by weight). By comparing the height profile before and after the KI-etching we can extract the actual gold thickness, which for us was $102.7 \mathrm{~nm}$.

We characterized each hemisphere in terms of radius of curvature, ellipticity $\epsilon$, and surface roughness or deviation from an ideal hemisphere. We define the latter as

$$
r_{q}=\sqrt{\frac{1}{N} \sum_{i}^{N}\left|z_{i}-f\left(x_{i}\right)\right|^{2}}
$$

where $f$ is the fit function, and $\epsilon=\left|R_{x}-R_{y}\right| /\left(R_{x}+R_{y}\right)$ as the relative difference of radii in arbitrarily chosen orthogonal axes and determine $R_{x}, R_{y}$ from fits. Figure S1(b) shows that hemispheres with smaller radii become more elliptical. Furthermore, the surface roughness also increases for these hemispheres (not shown in Figure S1(b)). This limits the the minimal radius of curvature to $>2.3 \mu \mathrm{m}$.

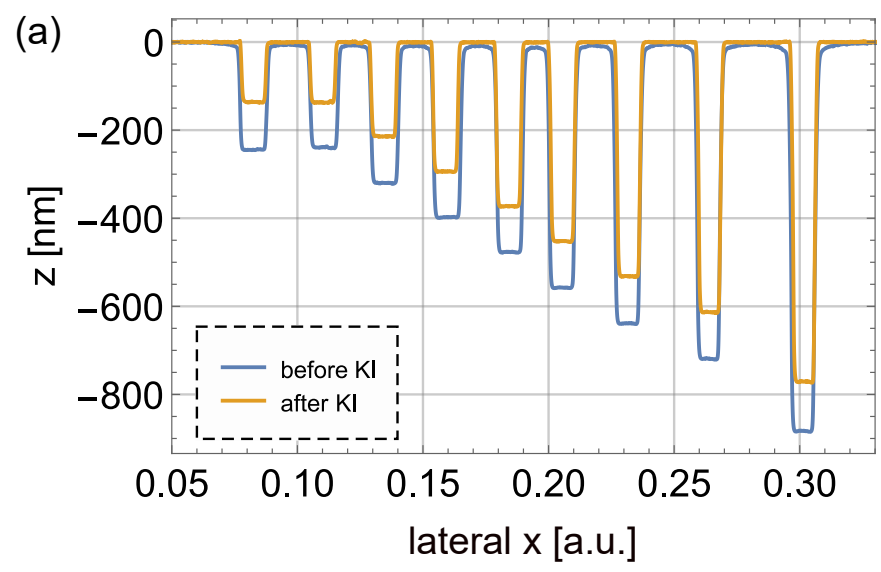

(b)

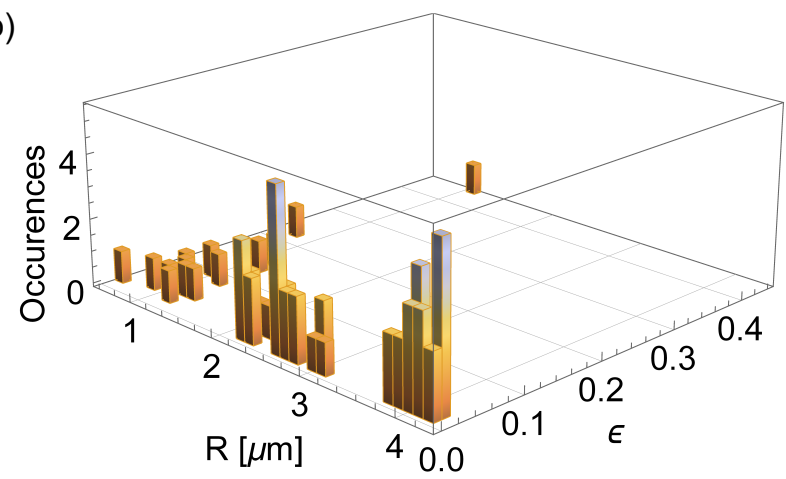

FIG. S1: Hemisphere fabrication. (a) Trace of the height profile etched using the FIB and scanned through a number of milled squares (different dose rate used for the squares). (b) The statistics show that for small radii of curvature below $2.3 \mu \mathrm{m}$, the hemispheres become more elliptical. In addition, while there are a few non-elliptical hemispheres with a tiny radius, all of these hemispheres suffer of a higher surface roughness.

\section{S2. SPIN SPEED CURVES PDMS SOLUTION}

We used the Sylgard 184 Silicone Elastomer kit as basis for the PDMS solution. The PDMS and curing agent were mixed in a 10:1 ratio. The solution was diluted using tert-Butyl alcohol (TBA). This allows us to achieve thin and uniform films while at the same time preventing swelling[1]. All substrates were plasma cleaned and substrate and solution were pre-baked at $40^{\circ} \mathrm{C}$ (TBA is a solid at room temperature). After spin coating, the PDMS films are cured at $125^{\circ} \mathrm{C}$ for $20 \mathrm{~min}$. To measure the spin speed curves we coated $\mathrm{Si}$ substrates with the solution at varying spin speeds and dissolved the PDMS film on about half of the Si chip using chemical etching (acetone and isopropyl alcohol). The chemical etching took place prior to the heat curing. Using a surface profiler (Bruker Dektak) we measured the 


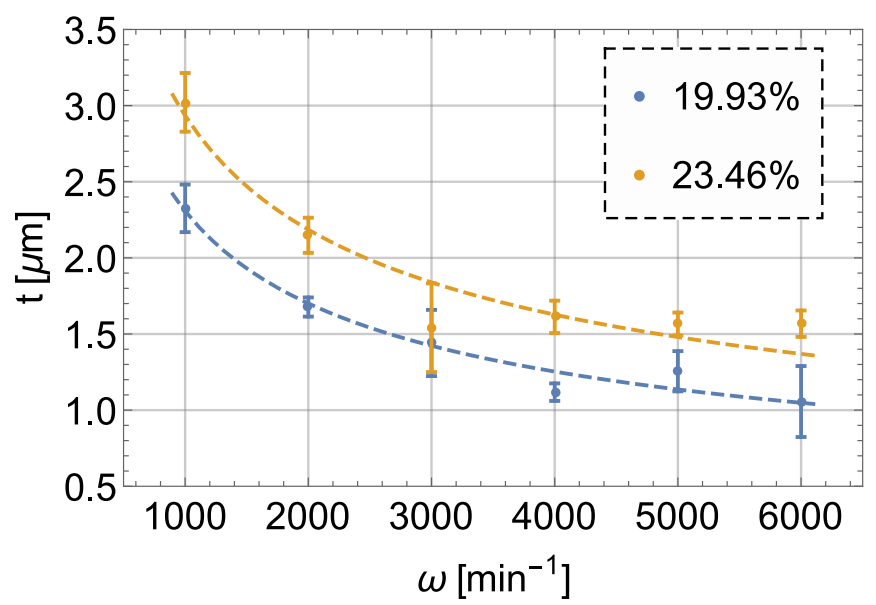

FIG. S2: Spin speed curves for different PDMS concentrations in TBA (error bars are $\sigma$ as defined in the text).

height of the PDMS film. We used a large stylus diameter $(12.5 \mu \mathrm{m})$ and minimal force $(1 \mathrm{mg})$ to reduce the pressure on the PDMS film, thereby getting an accurate measurement of the film thickness with a resolution of $1.1 \mathrm{~nm}$. We repeated the measurement at multiple locations on each chip. We define the total deviation of the mean $\bar{t}$ as

$$
\sigma=\sqrt{\sigma(\bar{t})^{2}+\overline{\sigma(t)}^{2}}
$$

where $\sigma(\bar{t})$ is the standard deviation of the mean of all measurements and $\overline{\sigma(t)}$ is the mean of the standard deviation of each measurement. Thus, the first term describes the roughness on large length scales, while the latter describes the local roughness. We fit the data with

$$
t(\omega)=\frac{t_{0}}{\omega^{\alpha}}
$$

For PDMS concentrations of $19.93 \%$ and $23.46 \%$ (by weight) and static dispense we find the experimentally derived constants $t_{0}=48.41 \mu \mathrm{m}, \alpha=0.441$ and $t_{0}=55.45 \mu \mathrm{m}, \alpha=0.425$, respectively. The corresponding spin speed curves are shown in Figure S2. Calculating the required spin speed allows us to hit the target thickness within $1 \%$. Note that for dynamic dispense the results become less accurate and reproducible. 


\section{S3. PHOTOGRAPHS OF THE DEVICE}

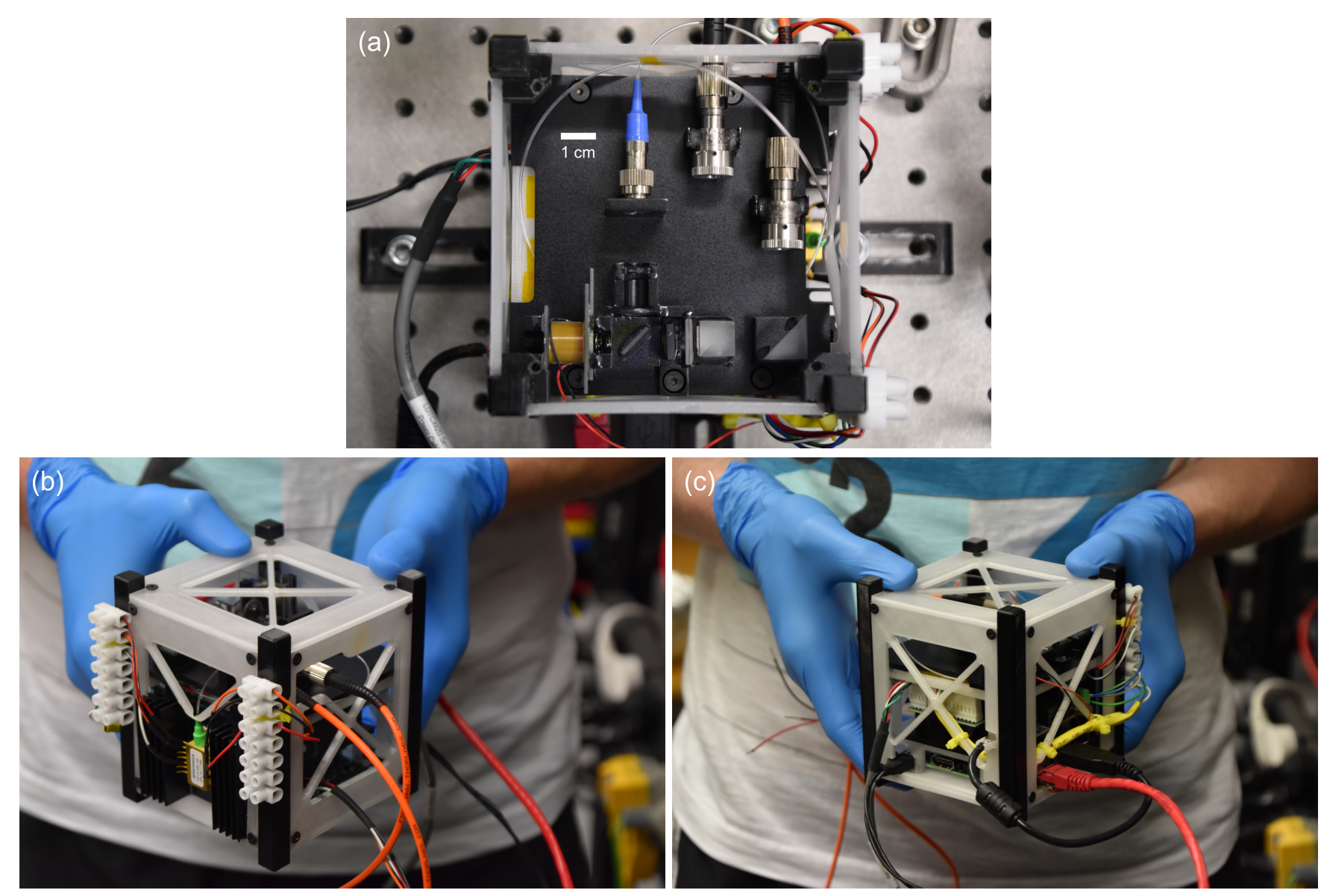

FIG. S3: Photographs of the device. (a) Top view optics platform. The complete device has an edge length of $10 \mathrm{~cm}$ (including 3D printed skeleton). (b),(c) Side-view. The skeleton is that of a $1 \mathrm{U}$ CubeSat, a picoclass satellite platform.

\section{S4. TRANSVERSE MODE SPACING}

The cavity modes (Hermite-Gaussian modes) are solutions to the Helmholtz equation in the paraxial approximation. The analytical solution for the resonant frequency of longitudinal mode $q$ and transverse mode $m, n$ is

$$
\nu_{m, n}^{q}=\frac{c}{2 L}\left(q+\frac{1+m+n}{\pi} \arccos \left(\sqrt{g_{1} g_{2}}\right)\right)
$$

with the cavity parameters $g_{i}=1-L / R_{i}$ depending on the radii of curvature of the spherical mirrors. For a flat mirror $g_{1}=1$, so the product of the cavity parameters in our case simplifies to $1-L / R$. The transverse mode spacing in wavelength space is thus

$$
\frac{c}{\nu_{0,1}^{8}}-\frac{c}{\nu_{0,0}^{8}}=22.7 \mathrm{~nm}
$$

which is much larger than the observed peak spacing in the spectrum (see main text). 


\section{S5. FDTD SIMULATIONS}

The finite-difference time-domain (FDTD) simulations were performed using Lumerical FDTD Solutions, a commercial-grade simulator based on the FDTD method 2]. To calculate the Purcell enhancement of a dipole emitter due to the substrate, we define the mirror as 9 stacks of alternating $\mathrm{SiO}_{2} / \mathrm{TiO}_{2}$ layers, with the $\mathrm{SiO}_{2}$ layer terminating the mirror. For the refractive indices we use the experimentally derived constants $\mathrm{n}_{\mathrm{SiO} 2}=1.521, \mathrm{n}_{\mathrm{TiO} 2}=2.135$ (see main text) and set the thickness to $92.9 \mathrm{~nm}$ for $\mathrm{SiO}_{2}$ and $66.2 \mathrm{~nm}$ for $\mathrm{TiO}_{2}$. To capture all dynamics we choose the mesh size much smaller than the size of the features. We define a dipole source at the $\mathrm{SiO}_{2}$-vacuum interface with an emission wavelength of $565 \mathrm{~nm}$ and its axis oriented in-plane with the dielectrics. The simulation uses PML (perfectly matched layer) boundary conditions, which are reflectionless or open boundaries. We sweep the distance $d$ of the dipole emitter to the dielectric stack and record the Purcell enhancement $\varepsilon$ at each position (see Figure S4(a)). As $d$ increases, the spontaneous emission rate oscillates and reaches unity (no enhancement or suppression) for $d \gg \lambda$.

We also model the plano-concave cavity by adding the hemispherical mirror to the simulation. Here, we leave the dipole at a fixed position and sweep the position of the hemispherical mirror. We record the intracavity electric field among other properties. The cavity is on resonance with the dipole when the intracavity power is maximal. Figure S4(b)-(d) show the electric field intensity as the mirror position is scanned: First, the cavity is detuned and the emission is suppressed (see Figure S4(b)). As the cavity length increases towards resonance, the intensity increases (see Figure S4(c)). Finally, at resonance the intensity is maximal (see Figure S4(d)).
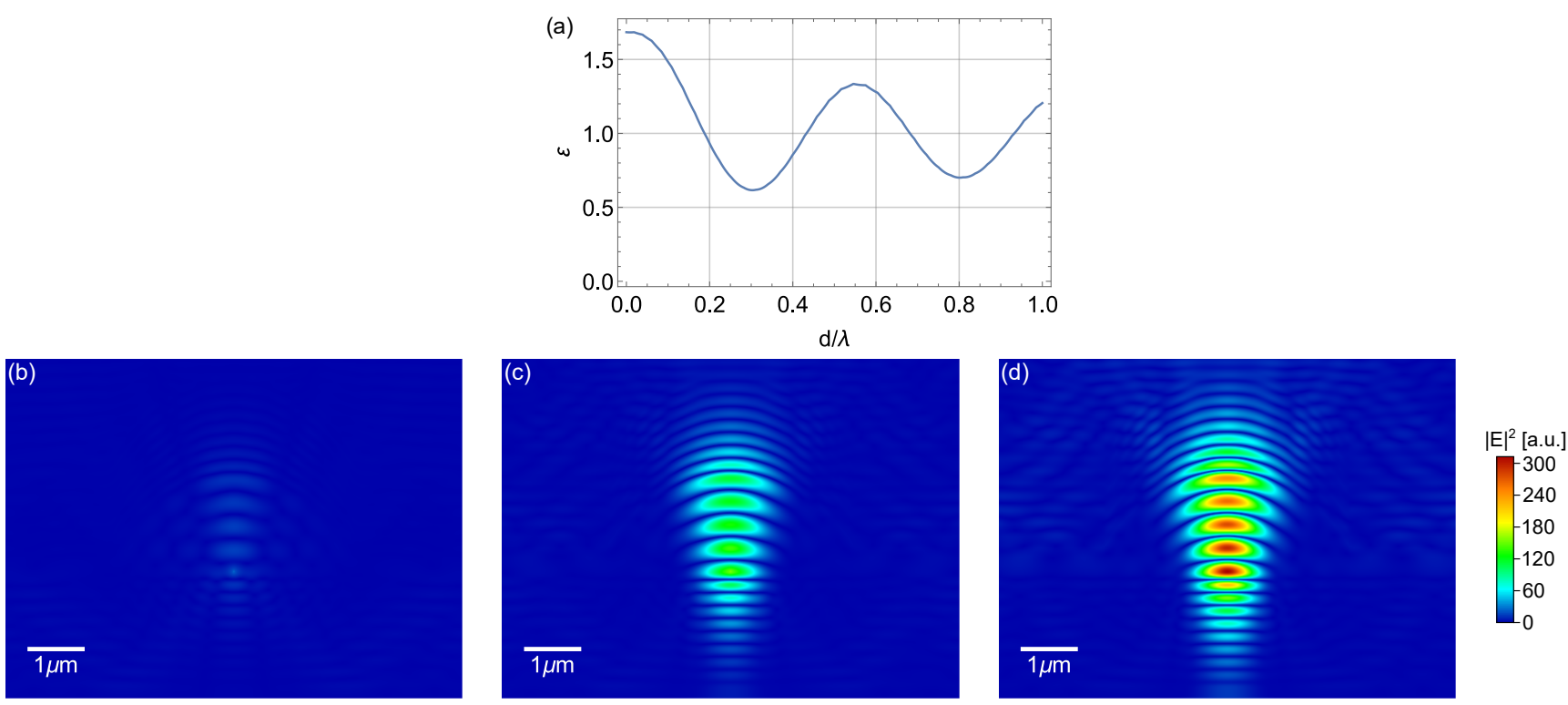

FIG. S4: FDTD simulations. (a) Purcell enhancement of a dipole emitter in close vicinity of a dielectric surface (mirror). As the distance $d$ of the dipole to the dielectrics increases, the spontaneous emission rate oscillates and reaches 1 (no enhancement) for $d \gg \lambda$. (b) Intracavity electric field in the plano-concave cavity, with the cavity detuned. As the cavity length is scanned towards resonance, the intracavity field increases (c) and reaches its maximum on resonance $(\mathrm{d})$.

\section{S6. QKD SIMULATIONS}

We also evaluate the performance of our SPS for quantum key distribution (QKD). Due to the lack of suitable SPSs the vast majority of QKD implementations use weak coherent states (WCSs). These are characterized by a low mean photon number (resulting in a low efficiency of the protocol) and a non-zero probability of emitting two or more photons at the same time. The multi-photon pulses contain information leaking to a potential eavesdropper. This can be reduced at the expense of sacrificing parts of the exchanged key. In comparison, an ideal single-photon source has an efficiency of unity and no multi-photon emission, so it always performs better than any protocol based on WCSs. We assess the performance of our single-photon source for the BB84 protocol[3] over a fiber channel with a loss of $0.21 \mathrm{~dB} / \mathrm{km}$ and realistic parameters from the experiment by Gobby, Yuan and Shields (GYS) 4 . It should be mentioned that such loss can only be achieved at telecom wavelengths, where single-photon emission from hBN has 
yet to be demonstrated, but for simplicity we still use all GYS parameters. The relevant metric is the extractable secret bit per sent signal. Moreover, we compare the results with an ideal SPS and the most common conventional QKD protocols: weak coherent and decoy states [5. The latter is the most efficient protocol that is publicly known. The simulations for weak coherent and decoy states assume a fixed mean photon number per pulse $\mu$, however, there is an optimal choice of $\mu$ for every distance.

The extractable secret bit per signal $[5$ for practical BB84-like protocols is

$$
S \geq q \max \left\{-Q_{\mu} f\left(E_{\mu}\right) h_{2}\left(E_{\mu}\right)+\Omega\left(1-h_{2}\left(e_{1}\right)\right), 0\right\}
$$

For BB84 $q=1 / 2$, because only in half of all cases the basis choice of sender and transmitter coincide. In the asymptotic limit of infinite qubits, however, $q$ can be chosen more efficiently (as high as 1 ). $Q_{\mu}$ and $E_{\mu}$ is the gain and quantum bit error ratio of the signal state, respectively. $f\left(E_{\mu}\right)$ is the error correction efficiency, which is in general dependent on the error ratio that has to be corrected. In the GYS experiment this is 1.22 for an error of $E_{\mu}=3.3 \%$. 4 . The binary Shannon entropy is defined as $h_{2}(p)=-p \log _{2}(p)-(1-p) \log _{2}(1-p) . \Omega$ is the fraction of detection events originating from single-photon events and $e_{1}$ is the error ratio on the single-photon state. The latter two are difficult to estimate, so one has to assume the worst case scenario that a photon-number splitting attack occurred on every multi-photon state and all errors originate from single-photon states. A better way to estimate these, however, is by using additional decoy states, whose statistics we measure, allowing us to find better bounds on $\Omega$ and $e_{1}[5$. In the asymptotic limit the fraction of decoy states can also be as low as 0 , so that the protocol is maximally efficient.

We implemented both protocols for a fiber channel of length $L$ with its transmission defined as

$$
\tau(L)=10^{-\alpha L}
$$

with $\alpha=0.21 \mathrm{~dB} / \mathrm{km}$, and for a free-space channel with its transmission (taking only diffraction losses into account) defined as

$$
\tau(L)=1-e^{-2 r^{2} / w^{2}(L)}
$$

with $r$ being the radius of the telescope of the receiver station and

$$
w(L)=\sqrt{w_{0}^{2}+\frac{L^{2} \lambda^{2}}{\pi^{2} w_{0}^{2}}}
$$

is the evolving beam width along $L . w_{0}$ is the radius of the transmitter telescope. Our simulations assume a $5 \mathrm{~cm}$ telescope for the transmitter and a $60 \mathrm{~cm}$ telescope for the receiver. The resulting transmissions through the fiber and free-space link are shown in Figure S5(a) and (b). As the link efficiency decreases, the received signal decreases as well, which results in a lower signal-to-noise ratio. At some point the detector dark counts contribute significantly to the quantum bit error ratio and thus the extractable secret bit per signal hits zero.

For our single-photon source the extractable secret bit per signal is given by

$$
S \geq q \max \left\{-f\left(E_{\mu}\right) h_{2}\left(E_{\mu}\right)+(1-\Delta)\left(1-h_{2}\left(\frac{E_{\mu}}{1-\Delta}\right)\right), 0\right\}
$$

where we again set $q=1$ and

$$
\Delta=\frac{g^{(2)}(0)}{\tau(L) \eta_{\operatorname{det}}(1-\eta)}
$$

is the probability that a multi-photon is emitted divided by the probability that any emitted photon is detected. $\eta$ is the quantum efficiency of the single-photon source and $\eta_{\text {det }}$ is the single-photon detector efficiency. For an ideal single-photon source of course $\Delta=0$. It should be mentioned that the sifted key rate (i.e. the rate that the receiver detects and this times $S$ is the secret key rate) scales with the mean photon number $\mu$ for the conventional protocols and with the quantum efficiency $\eta$ for our single-photon source. Again, for an ideal single-photon source $\eta=1$.

For the fiber link, at short and medium distances below $42 \mathrm{~km}$, our SPS outperforms both weak coherent and decoy states, while at long distances decoy states become more efficient (see Figure S5(c)). This is due to the fact that at long distances (i.e. high losses), multi-photon pulses harm the extractable secret bit disproportionately. Nevertheless, our SPS performs better than weak coherent states in each instance. Decoy state protocols can still achieve a finite secret key rate at large distances, because they can extract information from multi-photon states while at the same time defeating the photon-number splitting attack (multi-photon states dominate at long distances with high losses). A communication distance of $<42 \mathrm{~km}$ would be typical for metropolitan networks. The ideal SPS of course outperforms 

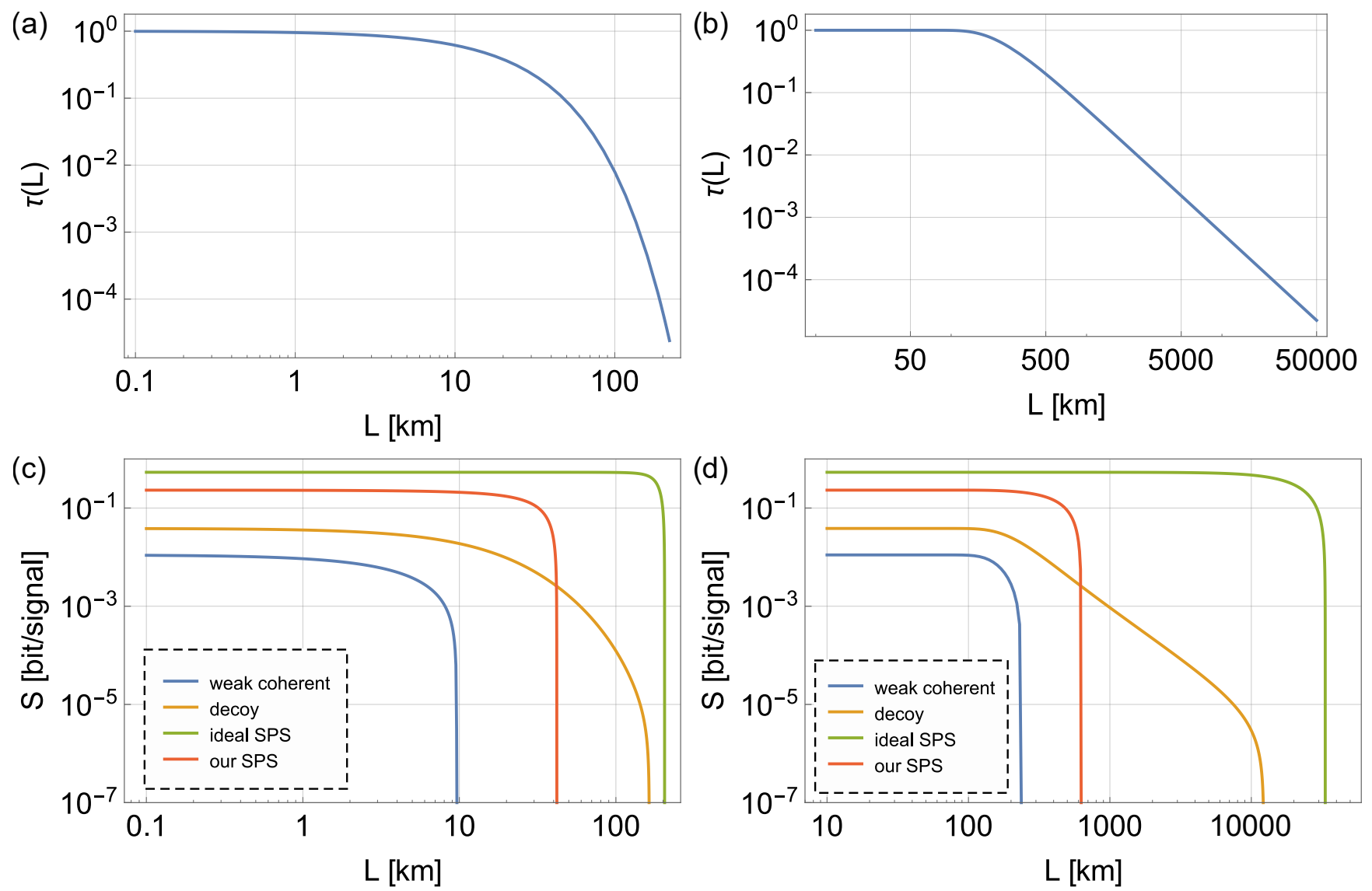

FIG. S5: Link transmission and QKD simulations. (a) for a fiber channel with losses of $0.21 \mathrm{~dB} / \mathrm{km}$ and (b) for a free-space channel with diffraction losses only, assuming a 5 and $60 \mathrm{~cm}$ telescope on the satellite and ground station, respectively. The wavelength is $565 \mathrm{~nm}$. (c) QKD for different photon sources for a fiber channel with $0.21 \mathrm{~dB} / \mathrm{km}$ loss. Our SPS outperforms weak coherent states at all distances and decoy state at short to medium distances up to $42 \mathrm{~km}$. (d) QKD for different photon sources for a free-space link. The transmitter assumes a $5 \mathrm{~cm}$ telescope, the receiver station a $60 \mathrm{~cm}$ telescope. Our SPS outperforms decoy states at distances up to $630 \mathrm{~km}$ and weak coherent states at all distances. For both channels, our single-photon source assumes $g_{0}^{2}=0.018$ and a quantum efficiency of $51.3 \%$.

all protocols and also our source at all distances. For space-to-ground links the loss is dominated by diffraction and atmospheric attenuation plays a role only in the lowest $10 \mathrm{~km}$. The simulations (see Figure $\mathbf{S 5}$ (d)) show that our source outperforms the decoy state protocol on distances up to $630 \mathrm{~km}$. For a comparison: The Micius satellite, which performed the first satellite-to-ground quantum key exchange, orbits at around $500 \mathrm{~km}[\underline{6}$. We note that the free-space loss channel assumes only diffraction losses and no other noise sources such as pointing errors, atmospheric loss and losses in the transmitter or receiver, which would change the result only marginally. The crossing point where our SPS and the decoy state protocol perform equally efficient for both channels is at a loss of $8.82 \mathrm{~dB}$.

Quantum emitters hosted by hBN can open unique opportunities for single-photon QKD applications, due to the large spread of optical transition lines [7. It is possible to choose a particular defect with a ZPL coinciding with one of the Fraunhofer lines (e.g. $\mathrm{H} \alpha$ ) in the solar spectrum. Narrow filtering around this line allows one to operate the QKD system at daylight, as the background from sunlight at that particular wavelength is suppressed [8]. Other color centers in solids have their ZPL fixed, so these cannot be used in this way.

[1] J. H. Koschwanez, R. H. Carlson, and Deirdre R. Meldrum, "Thin PDMS Films Using Long Spin Times or Tert-Butyl Alcohol as a Solvent," PLoS ONE 4, e4572 (2009).

[2] Lumerical Solutions, Inc., https://www.lumerical.com/products/fdtd-solutions/. 
[3] Nicolas Gisin, Grégoire Ribordy, Wolfgang Tittel, and Hugo Zbinden, "Quantum cryptography," Rev. Mod. Phys. 74, 145-195 (2002)

[4] C. Gobby, Z. L. Yuan, and A. J. Shields, "Quantum key distribution over $122 \mathrm{~km}$ of standard telecom fiber," Appl. Phys. Lett. 84, 3762-3764 (2004)

[5] Hoi-Kwong Lo, Xiongfeng Ma, and Kai Chen, "Decoy State Quantum Key Distribution," Phys. Rev. Lett. 94, 230504 (2005)

[6] Sheng-Kai Liao, Wen-Qi Cai, Wei-Yue Liu, Liang Zhang, Yang Li, Ji-Gang Ren, Juan Yin, Qi Shen, Yuan Cao, Zheng-Ping Li, Feng-Zhi Li, Xia-Wei Chen, Li-Hua Sun, Jian-Jun Jia, Jin-Cai Wu, Xiao-Jun Jiang, Jian-Feng Wang, Yong-Mei Huang, Qiang Wang, Yi-Lin Zhou, Lei Deng, Tao Xi, Lu Ma, Tai Hu, Qiang Zhang, Yu-Ao Chen, Nai-Le Liu, Xiang-Bin Wang, Zhen-Cai Zhu, Chao-Yang Lu, Rong Shu, Cheng-Zhi Peng, Jian-Yu Wang, and Jian-Wei Pan, "Satellite-to-ground quantum key distribution," Nature 549, 43-47 (2017).

[7] Toan Trong Tran, Christopher Elbadawi, Daniel Totonjian, Charlene J. Lobo, Gabriele Grosso, Hyowon Moon, Dirk R. Englund, Michael J. Ford, Igor Aharonovich, and Milos Toth, "Robust multicolor single photon emission from point defects in hexagonal boron nitride," ACS Nano 10, 7331-7338 (2016)

[8] D. J. Rogers, J. C. Bienfang, A. Mink, B. J. Hershman, A. Nakassis, X. Tang, L. Ma, D. H. Su, Carl J. Williams, and Charles W. Clark, "Free-space quantum cryptography in the H-alpha Fraunhofer window," Proc. SPIE 6304, 630417 (2006) 